${ }^{1}$ Centro Cardiovascular, Hospital Clínico Regional de Concepción: "Dr. Guillermo Grant Benavente". Concepción, Chile. 2Departamento de Cirugía, Facultad de Medicina, Universidad de Concepción. Concepción, Chile. ${ }^{3}$ Cirujano de Tórax, Servicio de Cirugía, Hospital Clínico Regional de Concepción: "Dr. Guillermo Grant Benavente". Concepción, Chile.

aPosbecada Cirugía General. IInterno Medicina, Facultad de Medicina, Universidad de Concepción. Concepción, Chile.

Recibido el 31 de julio de 2017 , aceptado el 27 de diciembre de

Correspondencia a: Roberto González Lagos Departamento de Cirugía, Facultad de Medicina, Universidad de Concepción, Janequeo esquina Chacabuco S/N, Concepción, Chile. Teléfono: (+56) (41) 2204883 rgonzalezlagos@udec.cl

\section{Traumatismo torácico: caracterización de hospitalizaciones durante tres décadas}

\author{
ROBERTO GONZÁLEZ L. ${ }^{1,2}$, ALEJANDRA RIQUELME U.,a, \\ ALBERTO FUENTES E. ${ }^{b}$, RENÉ SALDÍAS F. ${ }^{3}$, RODRIGO REYES M. ${ }^{2}$, \\ ENRIQUE SEGUEL S. ${ }^{1,2}$, ALECK STOCKINS L. ${ }^{1,2}$, ANDRÉS JADUE T. ${ }^{1}$, \\ JORGE CANALES Z. ${ }^{b}$, EMILIO ALARCÓN C. ${ }^{1,2}$
}

\section{Thoracic trauma. Experience of three decades}

Background: Trauma is the leading cause of death in young patients and thoracic trauma (TT) is responsible for $25-35 \%$ of trauma deaths. Aim: To describe and compare features, trauma severity indexes and morbidity of patients admitted for TT in the past three decades. Material and Methods: Review of a TT database, operative notes and medical records of patients. These were separated by decade of admission (1981-1990, 1991-2000, 2001-2010). TT characteristics were compared. Injury Severity Score (ISS), Revised Trauma Score (RTS-T) and Trauma Injury Severity Score (TRISS) were calculated. Results: A total of 3,068 TT were reviewed. In the 1981-1990 period, 604 cases of TT were registered (19.7\%), in the 1991-2000 period, 1,070 cases (34.9\%) and in the 2001-2010 period, 1,394 cases $(45.4 \%)(p<0.05)$. The ages of patients in each of these periods were $34.9 \pm 15.5,33.9 \pm 16.2$ and $35.7 \pm 18.2$ years respectively $(p<0.05)$. The proportion of patients aged 65 years or more were 6.6, 7.7 and $10.1 \%$ respectively, the proportion of females was 6.1, 9.4 and 12.0\%, respectively. The causing agents per decade were knife wounds in 51.5, 61.1 and 60.0\% of cases respectively, firearms in 2.5, 3.3 and $5.0 \%$ of cases respectively, multiple trauma in 13.9, 14.5 and 9.0\% respectively and morbidity in 18.7, 19.7 and $11.7 \%$, respectively. The ISS per decade was $11.9 \pm 6.5,12.9 \pm 6.9$ and $10.4 \pm$ 6.8 respectively. No significant difference were found in mortality $(1.5,3.0,2.0 \%$ respectively) or TRISS score (2.7, 3.2 and 3.8\% respectively). Conclusions: An increase in the number of hospital admission for TT has occurred in the last three decades, with an increase in the proportion of admissions of subjects aged 65 years or more, females and with firearm injuries.

(Rev Med Chile 2018; 146: 196-205)

Key words: Multiple Trauma; Thoracic Injuries; Thoracic Surgery; Trauma Severity Indices; Wounds and Injuries.
E traumatismo corresponde a la tercera causa de muerte en el adulto y la primera en menores de 40 años, siendo el traumatismo torácico (TT) responsable del 25-35\% de ellas. El TT se presenta en $10-15 \%$ de todos los traumatismos y hasta en $50 \%$ de los politraumatizados, afectando principalmente a hombres menores de 40 años. El TT se clasifica en contuso o penetrante, siendo su frecuencia dependiente del sitio geográfico. Usualmente no necesita alguna intervención quirúrgica mayor (80-85\%), siendo el tratamiento con pleurotomía y el tratamiento médico los más frecuentes ${ }^{1-5}$. Estudios aislados describen cambios en las características clínicas y epidemiología del TT a lo largo del tiempo.

Nuestro objetivo es describir y comparar las 
características, índices de gravedad de traumatismo (IGT) y morbimortalidad en pacientes hospitalizados por TT en las últimas 3 décadas.

\section{Material y Método}

Se realizó estudio descriptivo analítico en los pacientes hospitalizados por TT en el Hospital Clínico Regional "Dr. Guillermo Grant Benavente" de Concepción, Chile, tratados por el equipo de Cirugía Cardiotorácica, en el período comprendido entre enero de 1981 y diciembre de 2010. Las décadas fueron separadas en 3 períodos: 19811990, 1991-2000 y 2001-2010. La información se obtuvo a partir de protocolos prospectivos de TT realizados al momento de ingreso del paciente al hospital, revisión de base de datos del equipo, protocolos quirúrgicos y fichas clínicas con aprobación del Comité de Ética de la Facultad de Medicina de la Universidad de Concepción. Se ordenaron los datos mediante planilla Microsoft Excel $^{\oplus}$ y se utilizó programa SSPS $21^{\circledast}$ con función ANOVA de un factor para análisis estadístico con 3 variables cuantitativas y prueba chi cuadrado $\left(\chi^{2}\right)$ para variables cualitativas, además de estudio de regresión lineal de Pearson para análisis demográfico poblacional, calculando $R^{2}$, se consideró significativo $\mathrm{p}<0,05$. Se describe y compara número de hospitalizaciones, tasas según población regional, edad, género, características clínicas, mecanismos, tratamiento, morbimortalidad e índices de gravedad de traumatismo (IGT) y la relación de estos con la mortalidad.

Se aplicaron scores utilizados ampliamente en la literatura como: ISS (Injury Severity Score) ${ }^{6}$ que determina gravedad de acuerdo a lesión anatómica, RTS-T (Revised Trauma Score Triage) ${ }^{7}$ de acuerdo al estado o deterioro fisiológico al momento del ingreso (presión arterial sistólica, frecuencia respiratoria y escala de Glasgow) y el TRISS (Trauma Injurity Severity Score) ${ }^{8}$ score mixto, que engloba las dos anteriores y las relaciona con la edad del paciente y el mecanismo del traumatismo, determinando el riesgo de mortalidad ${ }^{5,9,10}$. Se consideró politraumatizado un ISS $>16$ puntos $^{9,11}$.

Se realizó búsqueda de registro de datos estadísticos poblacionales, en el Instituto Nacional de Estadística (INE) sobre población estimada entre los años 1981-2010 y resultados oficiales de censos de 1982-1992-2002-2012. Se calculó la tasa de hospitalizaciones por TT por 100.000 habitantes en Hospital Clínico Regional "Dr. Guillermo Grant Benavente" de Concepción, Chile según población de la Región del Biobío ${ }^{12-18}$.

\section{Resultados}

En el período 1981-2010 se hospitalizaron y fueron atendidos por nuestro equipo 3.095 pacientes con TT, de los cuales se excluyeron 27 $(0,9 \%)$ por ser menores de 15 años. Fue incluido un total de 3.068 pacientes. Al calcular la tasa anual por 100.000 habitantes de hospitalizaciones por TT en Hospital Clínico Regional "Dr. Guillermo Grant Benavente”, según la población regional, se observó un aumento significativo $(\mathrm{p}<0,05)$ desde 3,34 en año 1981 hasta 6,78 en 2010 (Figura 1).

Se encontró un aumento de la edad de los pacientes hospitalizados por TT, además de un aumento en la proporción de hospitalizaciones en pacientes $\geq 65$ años y de sexo femenino (Tabla 1 ).

Si bien se observó cambios en los traumatismos penetrantes y contusos en la década de 1991-2000, el TT penetrante predominó durante los 30 años. Con respecto al mecanismo del traumatismo, se encontró un aumento de la proporción de las hospitalizaciones por agresión y accidentes domésticos, con una disminución en los accidentes de tránsito y accidentes laborales (Tabla 2).

En relación al agente, se encontró un aumento del porcentaje de hospitalizaciones por lesiones por arma blanca y armas de fuego, además de una disminución de la proporción de TT por elementos contundentes y vehículos motorizados. Se observó también una disminución del porcentaje de politraumatizados hospitalizados con TT a lo largo de los años (Tabla 3).

Al observar exclusivamente las 1.856 (60,5\%) lesiones penetrantes, se observó un aumento significativo del porcentaje de hospitalizaciones por traumatismo torácico por arma de fuego, desde $4,6 \%$ en la década $1981-90,4,9 \%$ en la década 1991-2000, hasta 8,5\% en la década 2001-2010 $(\mathrm{p}<0,05)$.

Con respecto a los hallazgos y lesiones intratorácicas, se observó un aumento de la proporción de hospitalizaciones por heridas penetrantes cardiacas, con una disminución del porcentaje de hospitalizaciones por hemotórax no masivo, 

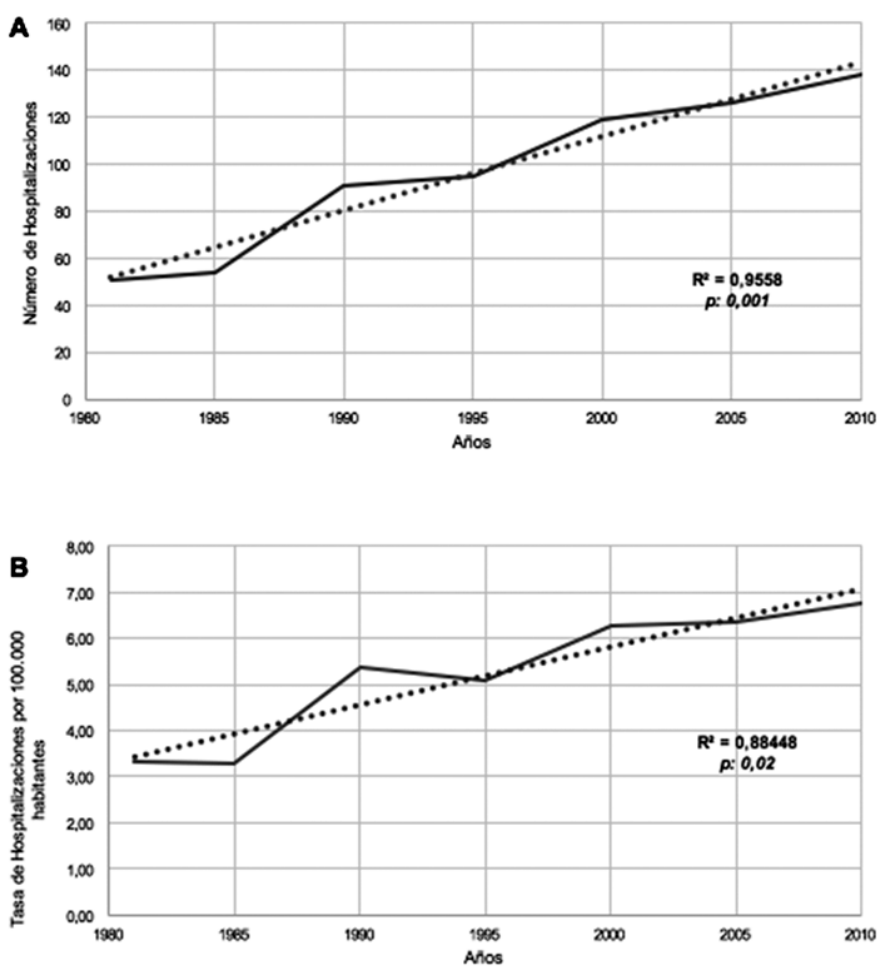

Figura 1. A: Número de pacientes hospitalizados por traumatismo torácico en Hospital Clínico Regional "Dr. Guillermo Grant Benavente" de Concepción, Chile durante 3 décadas; B: Tasas de hospitalizaciones por traumatismo torácico por 100.000 habitantes en Hospital Clínico Regional "Dr. Guillermo Grant Benavente" de Concepción, Chile según población de la Región del Biobío. neumotórax, fracturas costales y otros. Además, se observó una disminución de la proporción de hospitalizaciones por fracturas costales complicadas y tórax volante (Tabla 4 y 5). También se destaca una reducción del porcentaje de ingresos por TT asociados a lesiones craneoencefálicas o extremidades (Tabla 6).
En relación al tratamiento definitivo del TT, la mayoría de los pacientes $(70 \%)$ requirió tratamiento invasivo, si bien se observó una diminución de este durante las 3 décadas. Entre los pacientes que recibieron un tratamiento invasivo (2.159 pacientes), se trató de una pleurotomía en $78 \%$ y cirugía en $22 \%$ (Tabla 7 ).

Tabla 1. Número y características epidemiológicas en pacientes hospitalizados por traumatismo torácico en 3 décadas

\begin{tabular}{|c|c|c|c|c|c|}
\hline & $\begin{array}{c}\text { Total } \\
(3.068)\end{array}$ & $\begin{array}{c}1981-1990 \\
(604)\end{array}$ & $\begin{array}{c}1991-2000 \\
(1.070)\end{array}$ & $\begin{array}{c}2001-2010 \\
(1.394)\end{array}$ & $\mathbf{p}$ \\
\hline Número de pacientes & 3.068 & $604(19,7 \%)$ & $1.070(34,9 \%)$ & $1.394(45,4 \%)$ & 0,001 \\
\hline \multicolumn{6}{|l|}{ Características } \\
\hline Edad años (promedio) & $34,9 \pm 17,1$ & $34,9 \pm 15,5$ & $33,9 \pm 16,2$ & $35,7 \pm 18,2$ & 0,03 \\
\hline Mediana & 29 & 31 & 29 & 29 & \\
\hline Rango & 15-95 & $15-80$ & 15-95 & $15-95$ & \\
\hline$\geq 65$ años & $252(8,2 \%)$ & $40(6,6 \%)$ & $82(7,7 \%)$ & $141(10,1 \%)$ & 0,04 \\
\hline \multicolumn{6}{|l|}{ Sexo } \\
\hline Masculino & $2.762(90,0 \%)$ & $567(93,9 \%)$ & $969(90,6 \%)$ & $1.226(88,0 \%)$ & 0,001 \\
\hline Femenino & $306(10,0 \%)$ & $37(6,1 \%)$ & $101(9,4 \%)$ & $168(12,0 \%)$ & 0,001 \\
\hline
\end{tabular}


Tabla 2. Clasificación y mecanismo del traumatismo en pacientes hospitalizados por traumatismo torácico en 3 décadas

\begin{tabular}{|lcrrrc|}
\hline & $\begin{array}{c}\text { Total } \\
(\mathbf{3 . 0 6 8 )}\end{array}$ & $\begin{array}{c}\mathbf{1 9 8 1 - 1 9 9 0} \\
\mathbf{( 6 0 4 )}\end{array}$ & $\begin{array}{c}\mathbf{1 9 9 1 - 2 0 0 0} \\
\mathbf{( 1 . 0 7 0 )}\end{array}$ & $\begin{array}{c}\mathbf{2 0 0 1 - 2 0 1 0} \\
\mathbf{( 1 . 3 9 4 )}\end{array}$ & p \\
Clasificación & & & & & \\
Penetrante & $1.856(60,5 \%)$ & $335(55,5 \%)$ & $710(66,4 \%)$ & $811(58,2 \%)$ & 0,001 \\
Contuso & $1.212(39,5 \%)$ & $269(44,5 \%)$ & $360(33,6 \%)$ & $583(41,8 \%)$ & 0,001 \\
Mecanismo & & & & & \\
Agresión & $1.975(64,5 \%)$ & $350(58,0 \%)$ & $705(65,9 \%)$ & $920(66,0 \%)$ & 0,001 \\
Accidente de tránsito & $527(17,2 \%)$ & $121(20,0 \%)$ & $202(18,9 \%)$ & $204(14,6 \%)$ & 0,01 \\
Accidente doméstico & $216(7,0 \%)$ & $40(6,6 \%)$ & $47(4,4 \%)$ & $129(9,3 \%)$ & 0,001 \\
Accidente laboral & $82(2,7 \%)$ & $26(4,3 \%)$ & $29(2,7 \%)$ & $27 \quad(1,9 \%)$ & 0,01 \\
latrogénico & $30(1,0 \%)$ & $5(0,8 \%)$ & $19(1,8 \%)$ & $6(0,4 \%)$ & 0,003 \\
Autoagresión & $29(1,0 \%)$ & $3(0,5 \%)$ & $11(1,0 \%)$ & $15(1,1 \%)$ & $\mathrm{NS}$ \\
Otros mecanismo & $199(6,5 \%)$ & $59(9,8 \%)$ & $57(5,3 \%)$ & $83(6,7 \%)$ & 0,009 \\
\hline
\end{tabular}

NS: No estadísticamente significativo.

Tabla 3. Sitio, agente y tipo de traumatismo en pacientes hospitalizados por traumatismo torácico en 3 décadas

\begin{tabular}{|c|c|c|c|c|c|}
\hline & $\begin{array}{l}\text { Total } \\
(3.068)\end{array}$ & $\begin{array}{c}1981-1990 \\
(604)\end{array}$ & $\begin{array}{c}1991-2000 \\
(1.070)\end{array}$ & $\begin{array}{c}2001-2010 \\
(1.394)\end{array}$ & $\mathbf{p}$ \\
\hline \multicolumn{6}{|l|}{ Sitio del traumatismo } \\
\hline Vía pública & $2.339(76,2 \%)$ & $457(75,7 \%)$ & $871(81,4 \%)$ & $1.011(72,5 \%)$ & 0,001 \\
\hline Domicilio & $289 \quad(9,4 \%)$ & $49 \quad(8,1 \%)$ & 79 (7,4\%) & $161(11,6 \%)$ & 0,001 \\
\hline Otros & $440(14,4 \%)$ & $98(16,2 \%)$ & $120(11,2 \%)$ & $222(15,9 \%)$ & 0,001 \\
\hline \multicolumn{6}{|l|}{ Agente del traumatismo } \\
\hline Arma blanca & $1.802(58,7 \%)$ & $311(51,5 \%)$ & $654(61,1 \%)$ & $837(60,0 \%)$ & 0,001 \\
\hline Elemento contundente & $550(17,9 \%)$ & $141(23,3 \%)$ & $151(14,1 \%)$ & $258(18,5 \%)$ & 0,001 \\
\hline Vehículo motorizado & $520(17,0 \%)$ & $118(19,5 \%)$ & $199(18,6 \%)$ & $203(14,5 \%)$ & 0,005 \\
\hline Arma de fuego & 119 (3,9\%) & $15(2,5 \%)$ & $35 \quad(3,3 \%)$ & $69(5,0 \%)$ & 0,01 \\
\hline Vehículo no motorizado & $23 \quad(0,8 \%)$ & $11(1,8 \%)$ & $5 \quad(0,5 \%)$ & $7 \quad(0,5 \%)$ & 0,03 \\
\hline Otro agente & $54 \quad(1,8 \%)$ & $8(1,3 \%)$ & $26(2,4 \%)$ & $10 \quad(1,4 \%)$ & NS \\
\hline \multicolumn{6}{|l|}{ Tipo de TT } \\
\hline Aislado & $2.411(78,6 \%)$ & $468(77,5 \%)$ & $805(75,2 \%)$ & $1.138(81,6 \%)$ & 0,001 \\
\hline TT con lesiones asociadas & $657(21,4 \%)$ & $136(22,5 \%)$ & $265(24,8 \%)$ & $256(18,4 \%)$ & 0,001 \\
\hline Politraumatizado & $365(11,9 \%)$ & $84(13,9 \%)$ & $155(14,5 \%)$ & $126 \quad(9,0 \%)$ & 0,001 \\
\hline
\end{tabular}

NS: No estadísticamente significativo. TT: Traumatismo torácico.

Se cuantificó una disminución del porcentaje de morbilidad en pacientes hospitalizados por TT, principalmente aquellas atribuidas al traumatismo en sí. No se observó diferencias estadísticamente significativas en la mortalidad, salvo en los pacientes politraumatizados en los cuales si se observó un aumento del porcetaje de mortalidad a expensas de un aumento del TRISS desde $8,7 \%$ en la década 1981-90, 8,5\% en década 1991-2000, hasta 12,7\% en 2001-2010 ( $\mathrm{p}<0,05)$. Además se presentó una disminución significativa de la estadía hospitalaria por TT (Tabla 8). 
Tabla 4. Hallazgos y lesiones intratorácicas en pacientes hospitalizados por traumatismo torácico en 3 décadas

\begin{tabular}{|c|c|c|c|c|c|}
\hline & $\begin{array}{l}\text { Total } \\
(3.068)\end{array}$ & $\begin{array}{c}1981-1990 \\
(604)\end{array}$ & $\begin{array}{c}1991-2000 \\
(1.070)\end{array}$ & $\begin{array}{c}2001-2010 \\
(1.394)\end{array}$ & $\mathbf{p}$ \\
\hline Neumotórax & $1.698(55,3 \%)$ & $342(56,6 \%)$ & $641(59,9 \%)$ & $715(51,3 \%)$ & 0,001 \\
\hline Hemotórax no masivo & $1.656(54,0 \%)$ & $389(64,4 \%)$ & $619(57,9 \%)$ & $648(46,5 \%)$ & 0,001 \\
\hline Fracturas costales & $926(30,2 \%)$ & $253(41,9 \%)$ & $302(28,2 \%)$ & $371(26,6 \%)$ & 0,001 \\
\hline Enfisema subcutáneo & 765 (24,9\%) & $221(36,7 \%)$ & $326(30,5 \%)$ & $218(15,6 \%)$ & 0,001 \\
\hline Contusión pulmonar & $351(11,4 \%)$ & $131(21,7 \%)$ & $137(12,8 \%)$ & $83(6,0 \%)$ & 0,001 \\
\hline Penetrante cardíaca & $154(5,0 \%)$ & $18(2,9 \%)$ & $68 \quad(6,4 \%)$ & $68 \quad(4,9 \%)$ & 0,009 \\
\hline Lesión diafragmática & $90 \quad(2,9 \%)$ & $11(1,8 \%)$ & $37 \quad(3,5 \%)$ & $42(3,0 \%)$ & NS \\
\hline Hemotórax masivo & $87 \quad(2,8 \%)$ & $25(4,1 \%)$ & $28 \quad(2,6 \%)$ & $34 \quad(2,4 \%)$ & NS \\
\hline Fractura esternal & $55(1,8 \%)$ & $8 \quad(1,3 \%)$ & $7 \quad(0,7 \%)$ & $40 \quad(2,9 \%)$ & 0,001 \\
\hline Lesión parénquima pulmonar & $54 \quad(1,8 \%)$ & $13(2,2 \%)$ & $11(1,0 \%)$ & $30 \quad(2,2 \%)$ & NS \\
\hline Lesión grandes vasos torácicos & $31(1,0 \%)$ & $4 \quad(0,7 \%)$ & $10 \quad(0,9 \%)$ & $17(1,2 \%)$ & NS \\
\hline Neumotórax abierto & $23(0,8 \%)$ & $14(2,3 \%)$ & $4 \quad(0,4 \%)$ & $5 \quad(0,4 \%)$ & 0,001 \\
\hline Lesión vasos axilo-subclavios & $11(0,4 \%)$ & $0 \quad(0,0 \%)$ & $5 \quad(0,5 \%)$ & $6 \quad(0,4 \%)$ & NS \\
\hline
\end{tabular}

NS: No estadísticamente significativo.

Tabla 5. Fracturas costales y sus complicaciones en relación al total de pacientes hospitalizados por traumatismo torácico en 3 décadas

\begin{tabular}{|c|c|c|c|c|c|}
\hline & $\begin{array}{c}\text { Total } \\
(3.068)\end{array}$ & $\begin{array}{c}1981-1990 \\
(604)\end{array}$ & $\begin{array}{c}1991-2000 \\
(1.070)\end{array}$ & $\begin{array}{c}2001-2010 \\
(1.394)\end{array}$ & $\mathbf{p}$ \\
\hline 1 a 3 costillas & $495(16,1 \%)$ & $129(21,4 \%)$ & $129(12,1 \%)$ & $237(17,0 \%)$ & 0,001 \\
\hline 4 a 6 costillas & $336(11,0 \%)$ & $90(14,9 \%)$ & $132(12,3 \%)$ & $114 \quad(8,2 \%)$ & 0,001 \\
\hline$>6$ costillas & $95 \quad(3,1 \%)$ & $34 \quad(5,6 \%)$ & $41 \quad(3,8 \%)$ & $20 \quad(1,4 \%)$ & 0,001 \\
\hline Tórax volante & $154 \quad(5,0 \%)$ & $57 \quad(9,4 \%)$ & $76(7,1 \%)$ & $21 \quad(1,5 \%)$ & 0,001 \\
\hline Fracturas complicadas & $676(22,0 \%)$ & $205(33,9 \%)$ & $247(23,1 \%)$ & $224(16,0 \%)$ & 0,001 \\
\hline FC con complicación intratorácica & $665(21,7 \%)$ & $202(33,4 \%)$ & $246(23,0 \%)$ & $217(15,6 \%)$ & 0,001 \\
\hline FC con complicación extratorácica & $61 \quad(2,0 \%)$ & $10(1,7 \%)$ & $26(2,4 \%)$ & $25 \quad(1,8 \%)$ & NS \\
\hline
\end{tabular}

NS: No estadísticamente significativo. FC: Fracturas costales.

Tabla 6. Lesiones extratorácicas en pacientes hospitalizados por traumatismo torácico en 3 décadas

\begin{tabular}{|lccccc|}
\hline & $\begin{array}{c}\text { Total } \\
(\mathbf{3 . 0 6 8 )}\end{array}$ & $\begin{array}{c}\mathbf{1 9 8 1 - 1 9 9 0} \\
\mathbf{( 6 0 4 )}\end{array}$ & $\begin{array}{c}\mathbf{1 9 9 1 - 2 0 0 0} \\
\mathbf{( 1 . 0 7 0 )}\end{array}$ & $\begin{array}{c}\mathbf{2 0 0 1 - 2 0 1 0} \\
\mathbf{( 1 . 3 9 4 )}\end{array}$ & p \\
Craneo-encefálicas & $281(9,2 \%)$ & $81(13,4 \%)$ & $130(12,2 \%)$ & $70(5,0 \%)$ & 0,001 \\
Extremidades & $198(6,5 \%)$ & $37(6,1 \%)$ & $72(6,7 \%)$ & $60(4,3 \%)$ & 0,02 \\
Abdominales & $169(5,5 \%)$ & $29(4,8 \%)$ & $81(7,6 \%)$ & $88(6,3 \%)$ & NS \\
\hline Partes blandas & $90(2,9 \%)$ & $11(1,8 \%)$ & $18(1,7 \%)$ & $61(4,4 \%)$ & 0,001 \\
Fracturas de pelvis & $71(2,3 \%)$ & $16(2,7 \%)$ & $33(3,1 \%)$ & $22(1,6 \%)$ & 0,04 \\
\hline Faciales & $67(2,2 \%)$ & $9(1,5 \%)$ & $38(3,6 \%)$ & $20(1,4 \%)$ & 0,001 \\
\hline
\end{tabular}

NS: No estadísticamente significativo. 
Traumatismo torácico - R. González et al

Tabla 7. Tratamiento definitivo realizado en hospitalizados por traumatismo torácico en 3 décadas

\begin{tabular}{|lccccc|}
\hline & $\begin{array}{c}\text { Total } \\
\text { (3.068) }\end{array}$ & $\begin{array}{c}\mathbf{1 9 8 1 - 1 9 9 0} \\
\mathbf{( 6 0 4 )}\end{array}$ & $\begin{array}{c}\mathbf{1 9 9 1 - 2 0 0 0} \\
\mathbf{( 1 . 0 7 0 )}\end{array}$ & $\begin{array}{c}\mathbf{2 0 0 1 - 2 0 1 0} \\
\mathbf{( 1 . 3 9 4 )}\end{array}$ & p \\
\hline Tratramiento invasivo & $2.159(70,4 \%)$ & $446(73,9 \%)$ & $857(80,1 \%)$ & $864(62,0 \%)$ & 0,001 \\
Pleurotomía exclusiva & $1.685(54,9 \%)$ & $344(57,0 \%)$ & $633(59,2 \%)$ & $633(45,4 \%)$ & 0,001 \\
Cirugía & $474(15,5 \%)$ & $102(16,9 \%)$ & $224(20,9 \%)$ & $231(16,6 \%)$ & 0,01 \\
Médico exclusivo & $909(29,6 \%)$ & $159(26,1 \%)$ & $213(19,9 \%)$ & $530(38,0 \%)$ & 0,001 \\
\hline
\end{tabular}

Tabla 8. Morbilidad, reoperaciones, mortalidad y estadía hospitalaria en pacientes hospitalizados por traumatismo torácico en 3 décadas

\begin{tabular}{|c|c|c|c|c|c|}
\hline & $\begin{array}{c}\text { Total } \\
(3.068)\end{array}$ & $\begin{array}{c}1981-1990 \\
(604)\end{array}$ & $\begin{array}{c}1991-2000 \\
(1.070)\end{array}$ & $\begin{array}{c}2001-2010 \\
(1.394)\end{array}$ & $\mathbf{p}$ \\
\hline Morbilidad & $488(15,9 \%)$ & $113(18,7 \%)$ & $211(19,7 \%)$ & $164(11,7 \%)$ & 0,001 \\
\hline Atribuido a TT & $427(13,9 \%)$ & $104(17,2 \%)$ & $177(16,5 \%)$ & $146(10,4 \%)$ & 0,001 \\
\hline Atribuido a cirugía torácica & $54(1,8 \%)$ & $13(2,2 \%)$ & $26(2,4 \%)$ & $15(1,1 \%)$ & 0,03 \\
\hline Atribuido a cirugía extratorácica & $15(0,5 \%)$ & $1 \quad(0,2 \%)$ & $6 \quad(0,6 \%)$ & $8 \quad(0,6 \%)$ & NS \\
\hline Reoperaciones de cirugías torácicas & $32(1,0 \%)$ & $2(0,3 \%)$ & $11(1,0 \%)$ & $19(1,4 \%)$ & NS \\
\hline Mortalidad & $69(2,2 \%)$ & $9(1,5 \%)$ & $32 \quad(3,0 \%)$ & $28 \quad(2,0 \%)$ & NS \\
\hline TT aislado & $27(1,1 \%)$ & $3(0,6 \%)$ & $13(1,6 \%)$ & $11(1,0 \%)$ & NS \\
\hline TT con lesiones asociadas & $42(6,7 \%)$ & $6 \quad(4,4 \%)$ & $19(7,2 \%)$ & $17(6,6 \%)$ & NS \\
\hline Politraumatismo & $40(11,0 \%)$ & $6(7,1 \%)$ & $18(11,6 \%)$ & $16(12,7 \%)$ & 0,001 \\
\hline \multicolumn{6}{|l|}{ Estadía hospitalaria } \\
\hline Promedio (días) & $7,0 \pm 7,7$ & $8,4 \pm 6,6$ & $7,2 \pm 8,1$ & $6,4 \pm 7,7$ & 0,001 \\
\hline Medina & 5 & 7 & 5 & 5 & \\
\hline Rango & $1-150$ & $1-100$ & $1-147$ & $1-150$ & \\
\hline
\end{tabular}

NS: No estadísticamente significativo. TT: Traumatismo torácico.

Tabla 9. Índices de gravedad del traumatismo en pacientes hospitalizados por traumatismo torácico en 3 décadas

\begin{tabular}{|c|c|c|c|c|c|}
\hline & $\begin{array}{c}1981-2010 \\
(3.068)\end{array}$ & $\begin{array}{c}1981-1990 \\
(604)\end{array}$ & $\begin{array}{c}1991-2000 \\
(1.070)\end{array}$ & $\begin{array}{c}2001-2010 \\
(1.394)\end{array}$ & $\mathbf{p}$ \\
\hline \multicolumn{6}{|l|}{ Injury Severity Score (ISS) } \\
\hline Promedio & $11,3 \pm 6,8$ & $11,9 \pm 6,5$ & $12,9 \pm 6,9$ & $10,4 \pm 6,8$ & 0,001 \\
\hline \multicolumn{6}{|l|}{ Revised Trauma Score T (RTS-T) } \\
\hline Promedio & $11,7 \pm 1,1$ & $11,7 \pm 0,9$ & $11,7 \pm 1,1$ & $11,7 \pm 1,2$ & NS \\
\hline \multicolumn{6}{|c|}{ Trauma Injury Severity Score (TRISS) } \\
\hline Promedio (\% de mortalidad esperada) & 3,2 & 2,7 & 3,2 & 3,8 & NS \\
\hline Mortalidad observada & $2,2 \%$ & $1,5 \%$ & $3,0 \%$ & $2,0 \%$ & NS \\
\hline
\end{tabular}

NS: No estadísticamente significativo. 
Con respecto a los IGT, solo se encontró una disminución del ISS, sin cambios significativos en RTS-T ni TRISS. Tanto en el global como en cada uno de los períodos se observó una mortalidad menor a la esperada según el TRISS (Tabla 9).

\section{Discusión}

El TT es una importante causa de morbimortalidad, principalmente en pacientes menores de 40 años, por lo que es un tema relevante de estudio, ya que se trata de un problema de salud pública. Existen diferentes estudios descriptivos transversales de TT en las diferentes décadas, con los que se puede inferir ciertos cambios en las características clínicas y epidemiológicas de este tipo de traumatismo, pero hasta la fecha no existe un estudio que demuestre o confirme estos cambios ${ }^{1-5,19,20}$. El presente estudio busca mostrar una visión general de los cambios en las hospitalizaciones por TT en las últimas tres décadas.

Se observó un aumento significativo en la cantidad de hospitalizaciones por TT a lo largo de los años, como también de la tasa de hospitalizaciones por TT según la población regional, por lo que, el aumento de hospitalizaciones no solo se podría asociar a un incremento de la población regional, la cual aumentó, en el período estudiado, desde 1.496 .098 a 2.036 .443 habitantes ${ }^{12-18}$, sino que también, a una mayor expectativa de vida, a criterios más flexibles al momento de decidir la hospitalización, como también a un aumento de adultos mayores con TT.

El TT clásicamente se presenta en hombres jóvenes (61-94\%) con una edad promedio menor a los 40 años $(26,6-54,2 \%)^{1-5,19,21-23}$, nuestra serie demostró una disminución de la proporción de hospitalizaciones en hombres, con un aumento en las mujeres, la edad de ingreso y pacientes $\geq$ 65 años hospitalizados por TT a lo largo de estas 3 décadas.

La clasificación de TT penetrante o contuso y su frecuencia varía principalmente según ubicación geográfica. En diferentes estudios, principalmente estadounidenses y europeos, existe una predominancia de TT contusos, que alcanzan hasta $66-95,8 \%$, producidos por accidente de tránsito por vehículo motorizado $(22,8-70,9 \%)$ y caídas $(7,6-69,5 \%)^{3.4,20,24}$, siendo más frecuentes las lesiones penetrantes (34-65\%) en países latinoa- mericanos, producidas por arma blanca $(35-85 \%)$ $y$ armas de fuego (15-29\% $)^{5,22,25}$. En nuestra serie se observó un predominio, durante los 30 años, del TT penetrante sobre el contuso, asociándose a un incremento de la proporción de hospitalizaciones por agresiones, principalmente por armas blancas $\mathrm{y}$ armas de fuego, estas últimas duplicando su proporción. Junto a eso, se demuestra una disminución de la proporción de hospitalizaciones por accidentes de tránsito y laborales con elementos contundentes como caídas de nivel o altura. Sin embargo, se destaca un aumento de las lesiones contusas domésticas, lo que se podría explicar por el aumento de hospitalizaciones de adultos mayores, más propensos a caídas de nivel con lesiones de tipo contusas, siendo esta causa responsable de las hospitalizaciones por TT en adulto mayor hasta en $80 \%{ }^{26}$.

Se encontró un predominio de los TT aislados sobre los TT con lesiones asociadas y politraumatismos, estos últimos con una significativa disminución en su proporción en las últimas décadas a niveles menores a los observados en otras series $(26 \%)^{1,5}$, lo que supone una menor gravedad de los pacientes que son hospitalizados actualmente por TT. Cabe destacar que en otras series no determinaron el concepto de politraumatismo de acuerdo al ISS, por lo que en esas series el concepto de politraumatismo podría estar sobreestimado,11.

Las lesiones y hallazgos intratorácicos asociados más frecuentes a traumatismo son clásicamente, en la literatura, el hemotórax (20-56\%), neumotórax $(28-51 \%)$ y fracturas costales (30$49 \%$ ) y menos frecuente contusión pulmonar, lesión diafragmática, penetrante cardiaca, lesión de grandes vasos, fractura esternal, etc ${ }^{1-5,19,21,25,27,28}$. En nuestra serie se destacó principalmente una disminución proporcional de las hospitalizaciones por hemotórax no masivo, fracturas costales, tórax volante, contusión pulmonar y neumotórax abierto, asociado a un aumento en el porcentaje de hospitalizaciones por fractura esternal y penetrante cardiaca.

El TT se asocia frecuentemente a lesiones extratorácicas, llegando en algunas series a $26-35 \%$, principalmente de extremidades (12,7-19\%), abdominales $(8,3-13 \%)$, cráneo-encefálicas $(8,4-4,6 \%) y$, en menor medida, fractura de pelvis $(3,6 \%)$, raquimedular $(3 \%)$ y cabeza y cuello $^{1-4,29}$. En nuestra serie se destacó una disminución significativa del porcentaje de pacientes 
hospitalizados con lesiones extratorácicas, probablemente asociada a la menor gravedad de los TT hospitalizados, lo que se demuestra por una disminución de los politraumatismos, necesidad de cirugía y del ISS.

A pesar de la gravedad y morbimortalidad asociado al TT, solo 2,6-20\% de ellos necesitará alguna intervención quirúrgica mayor, siendo la pleurotomía exclusiva el principal método invasivo de tratamiento $(18,3-83,3 \%)$, seguida del tratamiento médico exclusivo $(37,6-79,1 \%)^{1-5,19,21,30-34}$. Estas diferencias se deben principalmente a la variabilidad del tipo de traumatismo (contuso o penetrante) en las diferentes series publicadas, con predominio de pleurotomía en las series latinoamericanas $(63-75 \%)^{35,36}$. La necesidad de cirugía extratorácica es habitual, encontrando una frecuencia del $18-20 \%$ en la literatura ${ }^{1-5,19-21}$.

A lo largo de las décadas se han producido cambios en el tratamiento del TT, si bien fue predominantemente invasivo $(62-80,1 \%)$, con necesidad de tratamiento quirúrgico mayor en $22 \%$ de ellas, se observó un aumento significativo de la proporción de tratamiento médico exclusivo y una disminución de la pleurotomía exclusiva y cirugía torácica, principalmente en la última década, probablemente asociado a la menor gravedad de las lesiones anatómicas, a la mayor edad de los pacientes hospitalizados por TT y a la facilidad de contar con estudios imageneológicos que facilitan el diagnóstico y la decisión hacia un tratamiento óptimo para el paciente.

La morbilidad asociada a TT es frecuente, alcanzando $8,7-35,5 \%$ y con una mortalidad que alcanza $1,8-10,3 \% \%^{1-5,35,36}$. La morbilidad en nuestro centro ha presentado una disminución significativa, probablemente por las causas antes comentadas, además de contar con mayores avances en los tratamientos que disminuyeron la estadía hospitalaria de nuestros pacientes. No se encontró diferencias en la mortalidad.

Los IGT corresponden a diferentes scores, que ayudan a determinar la gravedad y riesgo de mortalidad en pacientes con algún tipo de traumatismo, estableciendo su pronóstico, además de servir de base para líneas de investigación clínica, establecer aspectos epidemiológicos comparables entre distintos centros, ayudar a establecer programas de evaluación y control de calidad de atención médica, etc ${ }^{6-8}$. En nuestra serie, la mortalidad fue menor a la esperada según estos IGT en las diferentes décadas y en comparación con series latinoamericanas ${ }^{35,36}$. Durante el período solo se encontró una disminución de la gravedad anatómica de las lesiones (ISS) sin cambios significativo en el estado o deterioro fisiológico (RTS-T), lo que, junto a un aumento de la edad de los pacientes hospitalizados, principalmente un aumento en la proporción de aquellos $\geq 65$ años, podrían explicar la disminución de la morbilidad, necesidad de tratamiento invasivo, así como la mantención del TRISS y mortalidad a lo largo de estas 3 décadas.

Se destaca que solo se encontró un aumento significativo de la mortalidad en paciente politraumatizados, concomitante con un aumento del TRISS en este grupo, a pesar de una disminución de la proporción de hospitalización de pacientes con ISS > 16 durante estas 3 décadas, factores como el mecanismo contuso y el aumento de la edad, corresponden a factores de riesgo importantes de mortalidad y podrían explicar esto.

En conclusión, el TT se presenta por lo general en hombres jóvenes, producto, frecuentemente, de una lesión penetrante por agresión con arma blanca. Sin embargo, se han producidos cambios en las hospitalizaciones a lo largo de las tres últimas décadas. Se destaca, principalmente, un aumento del número de hospitalizaciones, junto a un aumento de las tasas según población regional, con un predominio de las lesiones penetrantes a lo largo de los 30 años, asociado a un aumento proporcional de las hospitalizaciones en mujeres y lesiones por arma de fuego, junto con un aumento de hospitalizaciones de paciente cada vez de mayor edad y de menor gravedad anatómica, además de una disminución de la proporción de politraumatizados, asociado a una menor necesidad de cirugías, morbilidad y estadía hospitalaria. No se observó cambios significativos en la mortalidad de la serie.

Agradecimientos: Agradecemos al Dr. Alberto Gyhra S. por iniciar este largo proyecto, por ser una constante inspiración y por su enorme generosidad.

\section{Referencias}

1. Demirhan R, Onan B, Oz K, Halazeroglu S. Comprehensive analysis of 4205 patients with chest trauma: a 
10-year experience. Interact Cardiovasc Thorac Surg 2009; 9 (3): 450-3.

2. Dongel I, Coskun A, Ozbay S, Bayram M, Atil B. Management of thoracic trauma in emergency service: Analysis of 1139 cases. Pak J Med Sci 2013; 29 (1): 58-63.

3. Al-Koudmani I, Darwish B, Al-Kateb K, Taifour Y. Chest trauma experience over eleven-year period at al-mouassat university teaching hospital-damascus: a restrospective review of 888 cases. J Cardiothorac Surg 2012; 7 (Apr): 35-42.

4. Kulshrestha P, Munshi I, Wait R. Profile of chest trauma in a level I trauma center. J Trauma 2004; 57 (3): 576-81.

5. Freixinet J, Beltrán J, Rodríguez P, Juliá G, Hussein M, Gil R, et al. Indicadores de gravedad en los traumatismos torácicos. Arch Bronconeumol 2008; 44 (5): 257 62 .

6. Baker SP, O’Neill B, Haddon W, Long W. The Injury Severity Score: a method for describing patients with multiple injuries and evaluating emergency care. J Trauma 1974; 14 (3): 187-96.

7. Champion H, Sacco W, Copes W, Gann D, Gennarelli T, Flanagan M. A revision of the Trauma Score. J Trauma 1989; 29 (5): 623-9.

8. Boyd C, Tolson M, Copes W. Evaluating trauma care: the TRISS method. Trauma Score and the Injury Severity Score. J Trauma 1987; 27 (4): 370-8.

9 Mica L, Rufibach K, Keel M, Trentz O. The risk of early mortality of polytrauma patients associated to ISS, NISS, APACHE II values and prothrombin time. J Trauma Manag Outcomes 2013;7 (May): 6.

10. Eid H, Abu-Zidan M. New injury severity score is a better predictor of mortality for blunt trauma patiens than the injury severity score. World J Surg 2015; 39 (1): 165-71.

11. Keel M, Trenz O. Pathophysiology of polytrauma. Injury 2005; 36 (6): 691-709.

12. Anuarios demografía y estadística vitals INE 1965-1999. Departamento de estadística e información de salud. En: http://www.deis.cl/anuarios-demografia-y-estadisticas-vitales-ine/

13. Resultados Oficiales Censo de población 1982. En: Instituto Nacional de Estadística. 1983. p. 11.

14. Resultados Oficiales Censo de Población 1992. En: Instituto Nacional de Estadística. 1993. p. 1, 13-16.

15. Informe Demográfico de Chile Censo 1992. En: Instituto Nacional de Estadística. 1993. p. 10.

16. Síntesis de Resultados Censo 2002. En: Instituto Nacional de Estadística. 2003. p. 9.

17. Censo 2002 Resultados Volumen I Población País-Región. En Instituto Nacional de Estadística. 2003. p. 45, 78.
18. Censo 2012 Resultados XVIII Censo de Población. En: Instituto Nacional de Estadística. 2013. p. 14, 55-60.

19. El-Menyar A, Lafiti R, Abdul-Rahman H, Zarour A, Tuma M, et al. Age an traumatic chest injury: a 3-year observational study. Eur J Trauma Emerg Surg 2013; 39 (4): 397-403.

20. Shorr R, Crittenden M, Indeck M, Hartunian S, Rodriguez A. Blunt thoracic trauma. Analysis of 515 patiens. Ann Surg 1987; 206 (2): 200-5.

21. Al-Eassa E, Al-Marashda M, Elsherif A, Eid H, Abu-Zidan F. Factors affecting mortality of hospitalized chest trauma patients in United Arab Emirates. J Cardiothorac Surg 2013; 8 (Mar): 57-63.

22. Sosa D, González J, Hernández J, Conde L, González P. Lesión penetrante y transfixiantes en los traumatismos abiertos del tórax. Rev Cubana Med Milit 2001; 30 (2): 73-80.

23. Freixinet J, Ramírez M, Gallardo G, Moreno P. Traumatismos torácicos. Arch Bronconeumol 2011; 47 (3): 9-14.

24. Liman S, Kuzucu A, Tastepe A, Ulasan G, Topcu S. Chest injury due to blunt trauma. Eur J Cardiothorac Surg 2003; 23 (3): 374-8.

25. Mefire A, Pagbe J, Fokou M, Nguimbous J, Guifo M, Bahebeck J. Analysis of epidemiology, lesions, treatment and outcome of 354 consecutive cases of blund and penetrating trauma to the chest in an African setting. S Afr J Surg 2010; 48 (3): 90-3.

26. Goodmanson N, Rosengart M, Bernato A, Sperry J, Peitzman A, Marshall G. Defining geriatric trauma: When does age make a difference? Surgery 2012; 152(4): 668-75.

27. O'connor JV, Adamski J. The diagnosis and treatment of non-cardiac thoracic trauma. J R Army Med Cops 2010; 156 (1): 5-14.

28. Sirmali M, Turut H, Topcu S, Gulhan E, Yazici U, Kaya S, et al. A comprehensive analysis of traumatic rib factures: morbility, mortality and management. Eur J Cardiothorac Surg 2003; 24 (1): 133-8.

29. Hill A, Fleiszer D, Brown R. Chest trauma in a Canadian urban setting - implications for trauma research in Canada. J Trauma 1991; 31 (7): 971-3.

30. Meredith J, Hoth J; Thoracic trauma: when an how to intervene. Surg Clin N Am 2007; 87 (1): 95-118.

31. Espinal R, Renán M, Romero R. Traumatismo torácico, análisis de 184 casos. Rev Med Honduras 1992; 60 (2): 75-80.

32. Ruiz J, Sánchez-Palencia A, Cueto A, Diéz J. Heridas torácicas: Revisión de 90 casos. Arch Bronconeumol 1999; 35 (2): 84-90.

33. Mackowski M, Barnett R, Harbrecht B, Miller K, 
Flanklin G, Smith J, et al. Damage control fot thoracic trauma. Am Surg 2014; 80 (9): 910-3.

34. Parry NG, Moffat B, Vogt K. Blunt thoracic trauma: recent advances and outstanding questions. Curr Opin Crit Care 2015; 21 (6): 544-8.

35. Jiménez C, Bautista S, Guzmán O, Soto J, Preciado N,
Rostro R, et al. Trauma de tórax. Experiencia de un año en el hospital de tercer nivel Hospital Civil Fray Antonio Alcalde. Rev Med MD 2012; 3 (4): 186-90.

36. Orozco S, Torres L, Otzoy H. Trauma torácico en la provincia de Cienfuegos. Estudio de tres años. Medisur 2014; 12 (1): 77-84. 